\title{
Metallographic Sectioning and Grinding Damage Analysis Through the Use of Color Metallography and Electron Backscatter Diffraction (EBSD)
}

\author{
G. M. Lucas*, G. F. Vander Voort*, P. T. Pinard**, M. Lagacé***, P. Hovington*** \\ * Buehler, Ltd., 41 Waukegan Rd. Lake Bluff, IL 60044 \\ ** Materials and Mining Eng. Dept., McGill University, 3610 University St., Montreal, Quebec, Canada H3A 2B2 \\ *** Hydro-Quebec Research Institute, 1800 Lionel-Boulet Boul., Varennes, Quebec, Canada J3X 1S1
}

This presentation is the second in a series derived from experiments to quantitatively determine the extent of damage caused by metallographic preparation steps through the use of color metallography with comparison to measurements using electron back scattered diffraction (EBSD). The previous presentation discussed damage cause by various sectioning methods and showed that EBSD could be successfully used to determine the depth of damage. However, the damage depth was not quantified at that time therefore it will be discussed in the first portion of the second presentation.

Two of the parameters that have the largest impact on the depth of damage are: the sectioning method used and the crystalline structure of the metal. Some of the more common sectioning methods include flame or plasma cutting, shearing, band sawing with or without cooling, dry abrasive cut-off sawing, wet abrasive cut-off sawing and precision cutting. Each of these methods produces a damage layer, some more considerable than others. Shearing produces deep mechanical damage but relatively little thermal damage. Flame or plasma cutting produces deep thermal damage but no mechanical damage. Band saw or hack saw lubricated but not cooled causes moderate thermal and mechanical damage. Dry abrasive cutting causes moderate to severe thermal damage. Wet abrasive cut-off saws cause minimal thermal and mechanical damage, while precision diamond saws cause the least thermal and mechanical damage. This is with the assumption these saws are being used correctly.

The second parameter that has an impact on the depth of damage is the crystalline structure of the metal itself. Three of the most common crystalline unit cell structures are face center cubic (FCC), body center cubic (BCC) and hexagonal close packed (HCP). Each of these will react differently to a sectioning method due to the differences in how the deform by slip or by twinning.

Specimens have been selected to represent these three crystalline structures stated above. The metals were sectioned by the methods stated above, prepared, tint etched and analyzed for sectioning damage. The same specimen were then re-prepared and analyzed by EBSD for sectioning damage and the results compared.

In the second portion of this presentation the damage caused by coarse grinding and fine grinding will be discussed. The same metals used for the sectioning experiments were ground and crosssectioned and prepared after each of the following grit of silicon carbide ( $\mathrm{SiC}$ ) paper and one diamond grinding disc (DGD): 60, 240 and $600 \mathrm{SiC}$; and, $55 \mu \mathrm{m}$ DGD (equivalent to 240 grit $\mathrm{SiC}$ ). The specimens were polished to a deformation free surface prior to being ground with the abrasive stated above. 


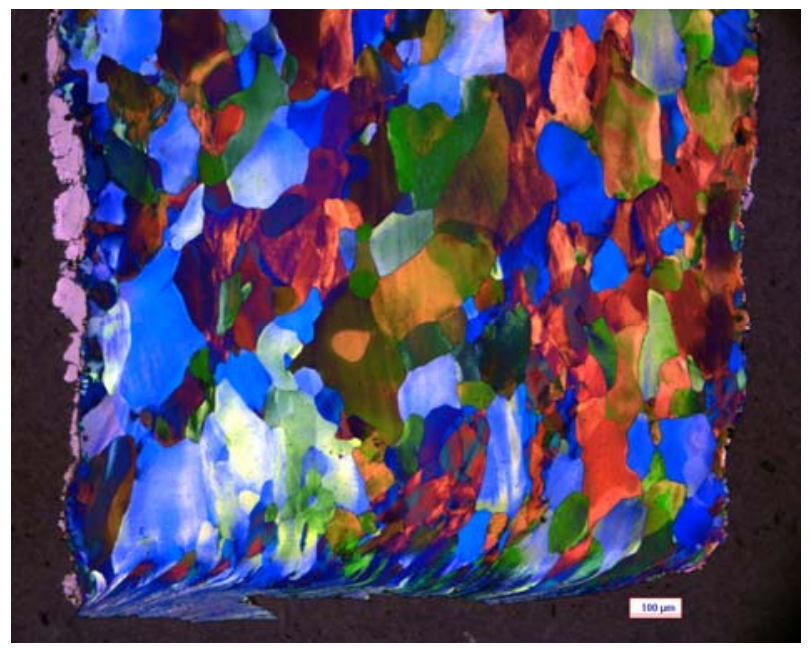

Fig. 1. 99.98 Fe sheared, etched with Klemm's I, $5 \mathrm{X}$ objective.

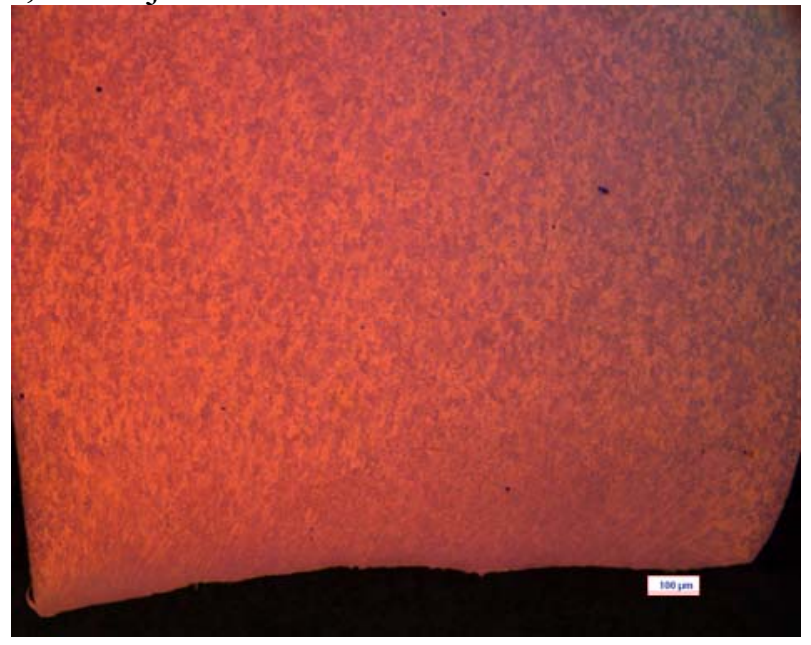

Fig. 3. CP Ti Sheared, as polished viewed in polarized light and sensitive tint, $5 \mathrm{X}$ objective

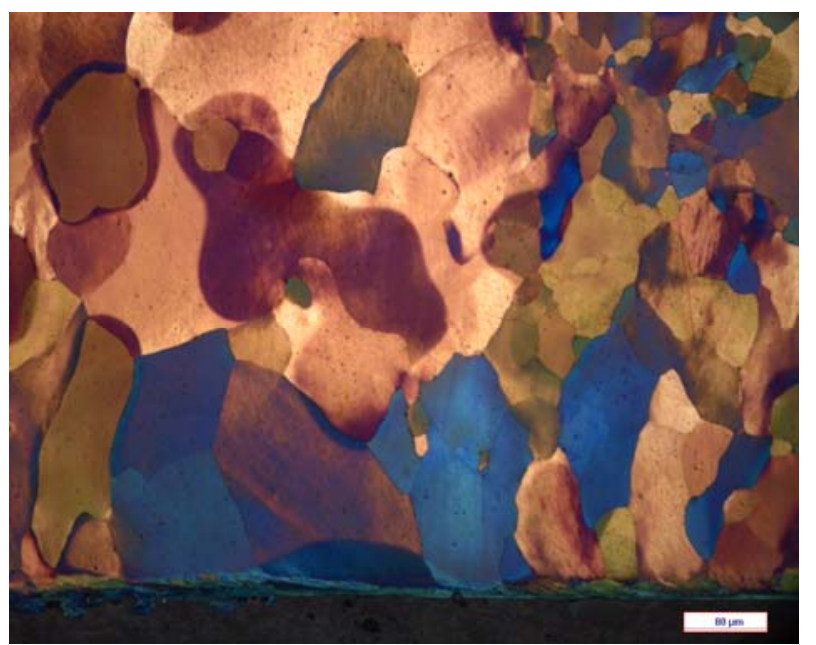

Fig. 2. 99.98 Fe Band saw, etched with Klemm's I, 10X objective.

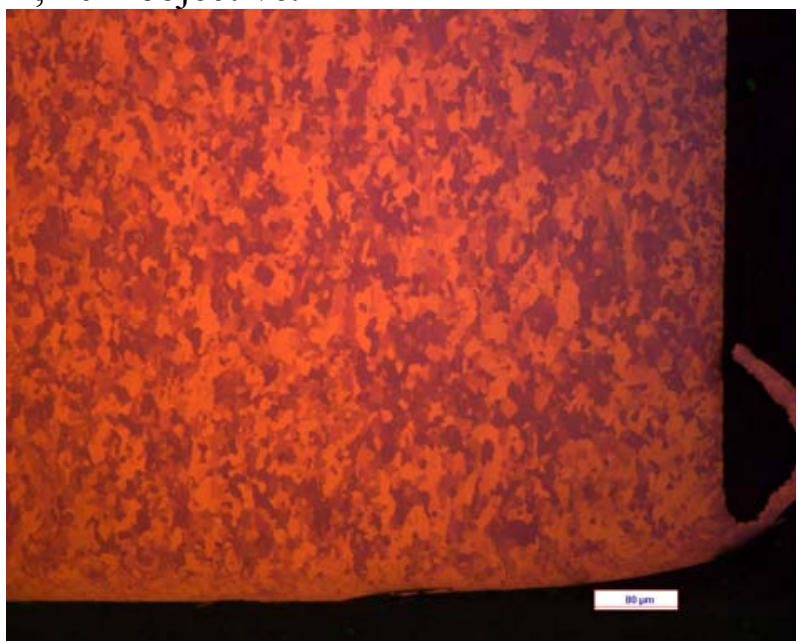

Fig. 4. CP Ti Band saw, as polished viewed in polarized light and sensitive tint, $5 \mathrm{X}$ objectiv
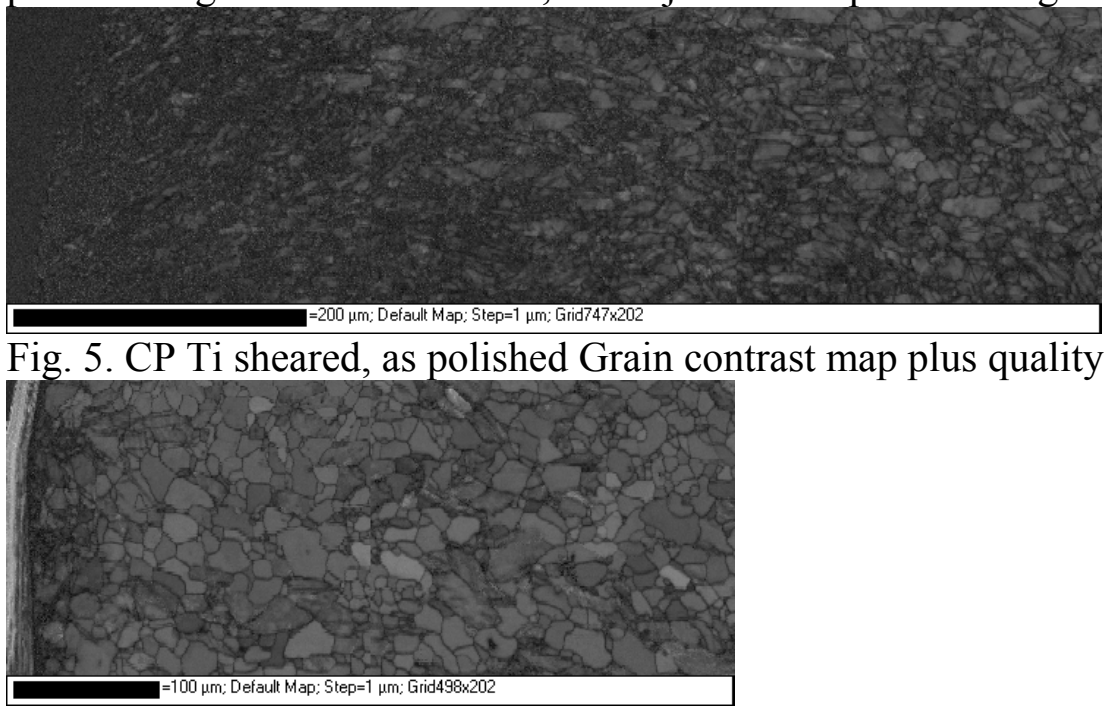

Fig. 6. CP Ti, Band saw cut, as polished Grain contrast map plus quality index map. 